\title{
Quasi-Biennial Modulation of the Solar Neutrino Flux: A "Telescope" for the Solar Interior
}

\author{
Loris D'Alessi $^{1}$, Antonio Vecchio ${ }^{1}$, Vincenzo Carbone ${ }^{1,2^{*}}$, Monica Laurenza ${ }^{3}$, Marisa Storini ${ }^{3}$ \\ ${ }^{1}$ Dipartimento di Fisica, Università della Calabria, Cosenza, Italy \\ ${ }^{2}$ Liquid Crystal Laboratory (INFM), Cosenza, Italy \\ ${ }^{3}$ INAF/IAPS-Roma, Via del Fosso del Cavaliere, Roma, Italy \\ Email: *vincenzo.carbone@fis.unical.it
}

Received February 18, 2013; revised March 21, 2013; accepted March 31, 2013

Copyright (C) 2013 Loris D'Alessi et al. This is an open access article distributed under the Creative Commons Attribution License, which permits unrestricted use, distribution, and reproduction in any medium, provided the original work is properly cited.

\begin{abstract}
An oscillating magnetic field deep within the solar radiative region can significantly alter the helioseismic g-modes. The presence of density gradients along g-modes, can excite Alfvén waves resonantly, the resulting waveforms show sharp spikes in the density profile at radii comparable with the neutrino's resonant oscillation length. This process should explain the observed quasi-biennial modulation of the solar neutrino flux. If confirmed, the coupling between solar neutrino flux and g-modes should be used as a "telescope" for the solar interior.
\end{abstract}

Keywords: Solar Neutrinos; Solar Cycle; Solar Interior

\section{Introduction}

Time variability of solar neutrino flux has been studied since the appearance of the first results of Homestake experiment [1]. In an early attempt to interpret the discrepancy between theoretical and observed flux, Sheldon [2] suggested a dependence of neutrino flux with solar activity, due to a time variable production rate of the neutrinos in the core of the Sun. The most famous evidence of the solar cycle is the time variation of sunspots number, with a characteristic period of about 11 years, extensively investigated in the past (see e.g., Ref. [3]). The occurrence of the solar cycle is related to the dynamo effect that generates the magnetic field of the Sun through the $\alpha-\omega$ process (the usual $\alpha$-effect coupled with the differential rotation) [4]. The spatial behaviour of the solar cycle is related to the latitudinal migration of magnetic structures toward the solar equator as the 11-year cycle goes on, thus generating the characteristic "butterfly diagram" in both space and time domain. Superimposed on these large-scale effects, the presence of small-scale apparently stochastic fluctuations is observed [5].

However, through the analysis of Homestake data Sakurai [6] showed the existence of a quasi-biennial periodicity both in the solar neutrino flux and in the sunspot number. In order to make a connection between the periodicity observed both in solar neutrino and sunspot data,

${ }^{*}$ Corresponding author.
Sakurai claimed that fluctuations of the core temperature, which is responsible of the $p p$ chain efficiency, should be at the origin of this quasi-biennial modulation [6]. However the analysis by Lanzerotti [7] carried on a set of data which cover a longer time period, exclude any connection between events in the core with the ones which occur in the photosphere. The results of analysis of Kamiokande data over the cycle 22 of the 11-yr solar cycle [8] showed that there is no correlation of the solar neutrino flux with sunspot numbers on 11-yr time scale.

Apart from the 11-year cycle, intra-cycle periodicities have been discovered in many solar activity proxies. The most prominently recognized periods are in the so called quasi-biennial oscillations (QBOs) range on time scales from 1.5 to 3.5 years [9-12]. This periodicity is better detected in correspondence of main cycle maxima and it suffers, as the 11-year cycle, of period length modulation [13]. Quite interestingly, corresponding QBOs have been found also in other contexts related to solar variability, as in solar wind fluctuations, interplanetary magnetic field intensity, galactic cosmic ray (CR) flux [14-17] energetic proton fluxes recorded in the interplanetary space [18] and in the solar rotation rate [19]. In they early work, Sakurai [6] invoked the presence of the quasi-biennial modulation for solar neutrino flux, in an attempt to solve the puzzle of missing neutrinos [20]. To date the puzzle has been solved in favour of neutrino flavour transformation 
[21], also implying a rest mass for neutrinos. Nevertheless, the origin of the biennial modulation of the solar neutrino flux and its interaction with the solar magnetic field are still debated [12,22-27].

These modulations should be induced by direct coupling of neutrino flux with solar magnetic field through neutrino magnetic moment. However, based on KamLAND data analysis constraint [28], assuming an upper bound for neutrino magnetic moment

$$
\mu_{v} \leq 10^{-12} \mu_{B}
$$

a neutrino oscillation length $\lambda_{\text {osc }} \approx 100-200 \mathrm{~km}$ and magnetic field fluctuation with amplitude $\delta_{B} \approx 50-100$ $\mathrm{kG}$, the deviation from the mean rate for SFP mechanism results to be of the order of $2.8 \times 10^{-2} \%$ [28]. This means that the coupling of neutrino's magnetic moment and magnetic field gives negligible effects. On these basis, the most reliable mechanism seems to be the modulation of the production rate of the nuclear reactions or the variation of physical parameters, mainly the density, at the solar core. In order to affect appreciably the neutrino flux, the density fluctuations have to satisfy both the following requests at the position of the MSW oscillation [29,30]:

1) The correlation length of these fluctuations has to be of the same order of neutrino oscillation length;

2) The fluctuations amplitude have to be at least of $\sim 1 \%$.

The most plausible mechanism, which in principle could originate fluctuations in matter density with the required properties, is the Alfvén/g-modes resonance [31]. The presence of density gradients along g-modes, can excite Alfvén waves resonantly, the resulting waveforms show sharp spikes in the density profile at radii comparable with the neutrino's resonant oscillation length.

Hence, the study of short-term periodicities of the solar cycle should lead to improve knowledge of the global properties of the Sun, with particular regard to solar neutrinos and energetic particle emission. In particular, the possible coupling neutrino-solar activity can help to understand the physical processes occurring in the solar deeper layers not accessible to helioseismic probing.

In the present paper we resume the study of the quasibiennial solar cycle (see [12,32-33]) by investigating the time evolution of two different datasets, through the empirical mode decomposition (EMD), with particular attention to the statistical significance of the analysis. We claim that the modulation can be the manifestation of the interaction of solar neutrino flux with Alfvén/g-mode resonance modulated by an oscillating magnetic field deep within the solar radiative region.

\section{The Neutrino Datasets}

In order to investigate the relationship between solar neutrinos and magnetic activity, we report the results of
EMD analysis carried out solar neutrino flux data recorded from the Homestake experiment (dataset $v_{H}$ ) (a total of 108 records from 1970 to 1994 [34]) and from the SAGE experiment (dataset $v_{S}$ ) (a total of 168 records from 1990 to 2008 [35]). The data from these two experiments cover a time window of $\sim 20 \mathrm{yr}$, passing through the maxima of two solar cycles (Cycles 21 and 22 for Homestake data and Cycles 22 and 23 for SAGE data). The EMD results for the two solar neutrino datasets have been compared with that obtained from the data of several solar cycle indicators: sunspot number (SN) and area (SA), flux of interplanetary protons in the energy range $0.50-0.96$ $\mathrm{MeV} /$ nucleon measured by the charged particles measurements experiment (channel P2) aboard the IMP8 spacecraft (P2) and cosmic ray intensity measured by the Rome neutron monitor with cutoff rigidity of $6 \mathrm{GV}$ $(\mathrm{NM})^{1}$.

\section{The Empirical Mode Decomposition}

The periodicities and their relative amplitudes have been identified through the EMD, a technique developed to process nonstationary data [36] and successfully applied in different contexts, e.g. [37,38]. In the EMD framework, a time series $X(t)$ is decomposed into a finite number of oscillating intrinsic mode functions (IMF) as

$$
X(t)=\sum_{j=0}^{m-1} C_{j}(t)+r_{m}(t)
$$

The IMFs $C_{j}(t)$ represent a set of basis functions obtained from the dataset under analysis by following the "sifting" procedure described by Huang et al. [36]. This procedure starts by identifying local minima and local maxima of the raw signal $X(t)$. The envelopes of maxima and minima are then obtained through cubic splines and the mean between them, namely $m_{1}(t)$, is calculated. The differences between the raw time series and the mean series $h_{1}(t)=X(t)-m_{1}(t)$, represents an IMF only if it satisfies two criteria: 1$)$ the number of extremes and zero crossings does not differ by more than one; 2 ) at any point, the mean value of the envelopes defined by the local maxima and the local minima is zero.

\section{Results and Discussion}

The EMD represents a powerful tool to study the solar QBOs and highly nonstationary signal. Since these oscillations are high during the activity maxima $[9,11,13]$ and their frequency is not constant from a cycle to another [13] the EMD is more suitable than the classical Fourier and wavelet analysis, to properly identify the QBOs. In fact it is well known that, in presence of nonstationary

${ }^{1} \mathrm{SN}$ and SA data at: http://solarscience.msfc.nasa.gov/SunspotCycle shtml; P2 data at: http://sdwww.jhuapl.edu/IMP/imp_cpme_data.html; CR data at: http://www.fis.uniroma3.it/svirco/. 
signals, the Fourier power spectrum, as well as the time integrated Wavelet spectrum, detect broader and lowered peaks. Since the Fourier transform looks for a global frequency and does not take into account possible period modulations, an underestimation of the contribution of the QBOs could occur. For each dataset, the QBO contribution to the original signal has been isolated through partial sum of IMFs oscillating with time scales in the range $1.4 \mathrm{yr} \leq \tau_{i} \leq 4 \mathrm{yr}$, where $\tau_{i}$ denotes a typical average period for the $i$-th IMF. The QBO contribution from the Homestake is shown in Figure 1 toghether with the quasi-biennial signals of P2 and NM, while in Figure 2 the QBO signal extracted from SAGE data is shown toghether with the QBOs of sunspot data. As a reference, the time history of the sunspot area for the period of reference is reported in the lower panel.

After properly identifying the QBO components through the EMD from the different indicators, we compare them by evaluating Pearson's correlation coefficient. For each correlation coefficient, a confidence level of 95\% is derived both through Fisher's transformation $\left(\Delta r_{F}\right)$ and bootstrap methods $\left(\Delta r_{\text {boot }}\right)$. Finally an estimation of the p-value (i.e. the probability to obtain by chance a correlation coefficient greater then that observed) is given by random phases method $\left(P_{R P}\right)$ [39]. Results demonstrate that the correlation is stronger around the solar cycle maxima where the QBO amplitudes are higher. In particular, the QBOs isolated from Homestake data are in phase with particles data around the maxima of cycle 21 and 22, while QBOs isolated from SAGE data seem to be correlated with those of sunspot data near the maxima of cycle 22 and 23. This correlation is significant even extending the time window to 11-yr starting from mid-1991. In Table 1 are shown the results of the correlative analysis.

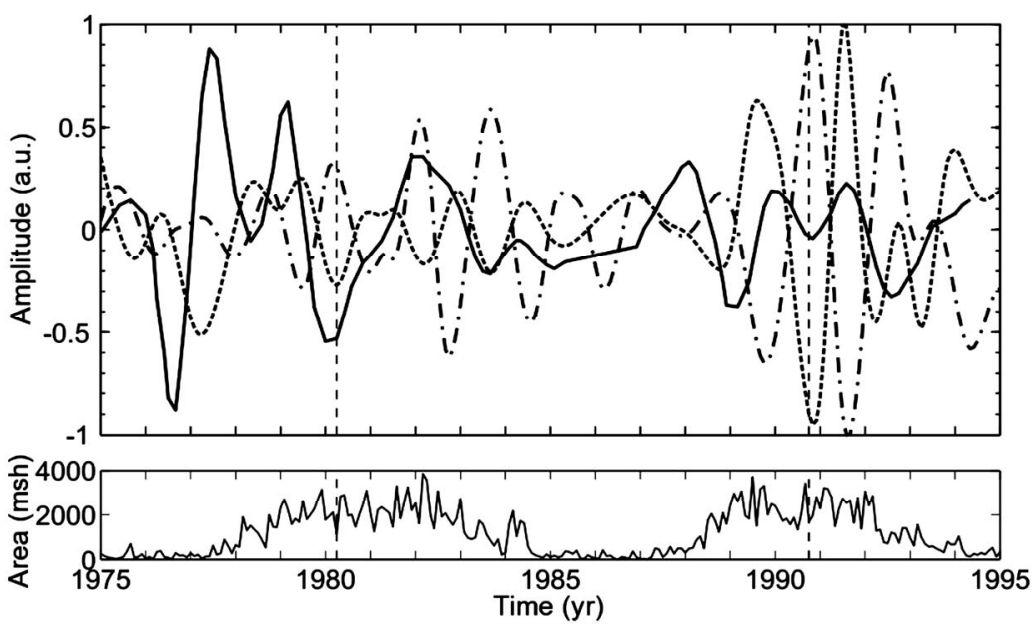

Figure 1. Upper panel: QBO isolated from Homestake data (solid line), P2 proton flux (dotted line) and galactic CRs (dash-dotted line). Lower panel: Time history of the sunspot areas for the period of reference (in unit of millionths of a solar hemisphere). Dashed vertical lines correspond to maxima of solar cycles.

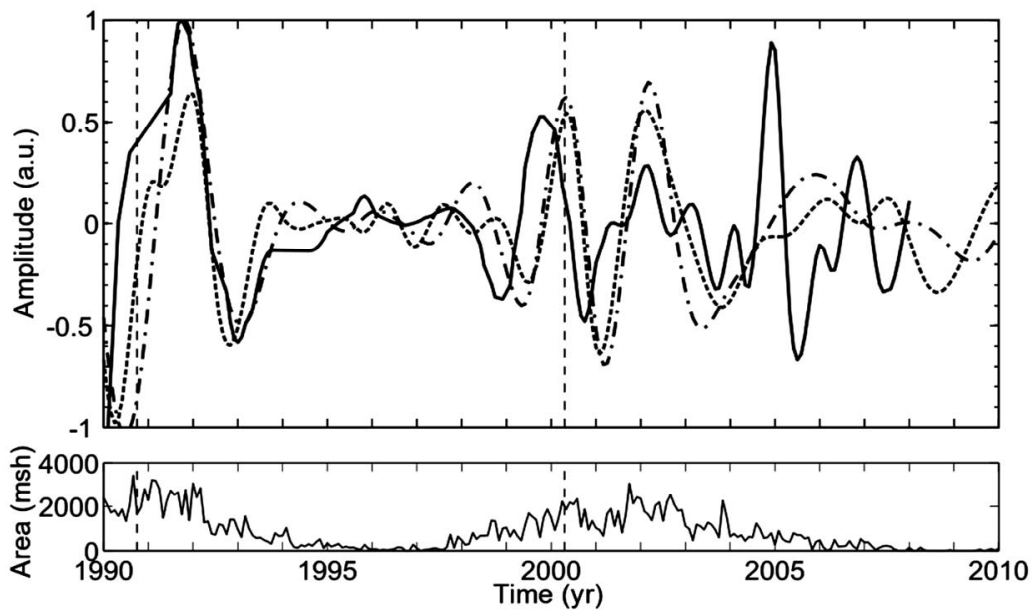

Figure 2. Upper panel: QBO isolated from SAGE data (solid line), sunspot area (dotted line) and sunspot number (dash-dotted line). Lower panel: Time history of the sunspot areas for the period of reference (in unit of millionths of a solar hemisphere). Dashed vertical lines correspond to maxima of solar cycles. 
Notes on tables contents. In Tables 1-3 are reported, for different couples of QBOs extracted from datasets $X$ and $Y$, the corresponding Pearson correlation coefficient $r$ evaluated in the period indicated in the caption. $\Delta r_{F}$ and $\Delta r_{\text {boot }}$ represent the $95 \%$ confidence intervals for the correlation coefficient from Fisher's and bootstrap tests, respectively. $P_{R P}$ indicates the probability, calculated through the random phases test, to obtain values greater than $r_{X Y}$ due to chance.

\section{The Magneto-Gravity Modes}

The observed correlation between solar quasi-biennial cycle and solar neutrino flux fluctuations on quasi-biennial time scales could represent a direct observation of instabilities induced by quasi-biennial dynamo effects in the deeper regions of solar radiative zone. The theory of coupling between large scale magnetic fields and solar matter has been investigated by Burgess et al. [31]. In particular, in the presence of backgroud magnetic fields of reasonable intensity, density gradients allows g-modes to excite Alfvén waves resonantly, causing mode energy to be funnelled along magnetic field lines away from the solar equatorial plane.

Magneto-gravity waves are described by the usual compressible, ideal magnetohydrodynamic equations,

Table 1. Results of correlative analysis for Homestake, energetic proton and cosmic ray data for $5 \mathrm{yr}$ around maxima of cycle 21 (1980.25).

\begin{tabular}{ccccc}
\hline$X-Y$ & $r$ & $\Delta r_{F}$ & $\Delta r_{\text {boot }}$ & $P_{R P}$ \\
\hline$v_{H}$-P2 & 0.96 & {$[0.90,0.98]$} & {$[0.91,0.98]$} & 0.01 \\
$v_{H}$-NM & -0.90 & {$[-0.96,-0.75]$} & {$[-0.95,-0.78]$} & 0.06 \\
P2-NM & -0.98 & {$[-0.99,-0.95]$} & {$[-0.99,-0.97]$} & 0.01 \\
\hline
\end{tabular}

Table 2. Results of correlative analysis for Homestake, energetic proton and cosmic ray data for $5 \mathrm{yr}$ around maxima of cycle 22 (1990.75).

\begin{tabular}{ccccc}
\hline$X-Y$ & $r$ & $\Delta r_{F}$ & $\Delta r_{\text {boot }}$ & $P_{R P}$ \\
\hline$v_{H}$-P2 & -0.92 & {$[0.82,0.97]$} & {$[0.82,0.97]$} & 0.03 \\
$v_{H}$-NM & 0.93 & {$[-0.99,-0.97]$} & {$[-0.99,-0.98]$} & 0.01 \\
P2-NM & -0.99 & {$[-0.97,-0.80]$} & {$[-0.96,-0.85]$} & 0.03 \\
\hline
\end{tabular}

Table 3. Results of correlative analysis for SAGE, sunspot number and area for 11 yr starting from mid-1990.

\begin{tabular}{ccccc}
\hline$X-Y$ & $r$ & $\Delta r_{F}$ & $\Delta r_{\text {boot }}$ & $P_{R P}$ \\
\hline$v_{S}$-SA & 0.58 & {$[0.46,0.69]$} & {$[0.45,0.69]$} & $<0.01$ \\
$v_{S}$-SN & 0.67 & {$[0.56,0.75]$} & {$[0.53,0.78]$} & $<0.01$ \\
SA-SN & 0.89 & {$[0.85,0.92]$} & {$[0.86,0.92]$} & $<0.01$ \\
\hline
\end{tabular}

namely the continuity equation for mass density

$$
\frac{\partial \rho}{\partial t}+\nabla \cdot(\rho \boldsymbol{v})=0
$$

the momentum equation with the gravity term

$$
\rho\left[\frac{\partial \boldsymbol{v}}{\partial t}+(\boldsymbol{v} \cdot \nabla) \boldsymbol{v}\right]=-\nabla P+\rho \boldsymbol{g}+\frac{1}{4 \pi}[(\nabla \times \boldsymbol{B}) \times \boldsymbol{B}]
$$

and the magnetic field induction equation

$$
\frac{\partial \boldsymbol{B}}{\partial t}=\nabla \times(\boldsymbol{v} \times \boldsymbol{B})
$$

The system of equations can be closed by relating the pressure $P$ to the mass density through an energy equation

$$
\frac{\mathrm{d} P}{\mathrm{~d} t}-\gamma \frac{P}{\rho}\left(\frac{\mathrm{d} \rho}{\mathrm{d} t}\right)=-(\gamma-1) Q
$$

where $\mathrm{d} / \mathrm{d} t$ is the total time derivative, $\gamma$ is the ratio of heat capacities, and $Q$ is the sum of all energy density sources and losses, such as heat conductivity, viscosity and ohmic dissipation.

Assuming an equilibrium situation where the velocity field and current density are both zero, Equations (3)-(6) are linearized by using low-frequency approximation, in order to filter out the pressure p-modes, and an exponenttial density profile. A plane geometry with a local gravity directed along the $z$-axis and the background magnetic field along the $x$-axis is used. Background quantities depend on $z$, and a standard mass-density profile coming from solar models $\rho_{0}=\rho_{c} \exp (-z / H)$ is assumed (here the density at the solar centre $\rho_{c}$ and the density height-scale $H$ are constant) [29]. All fluctuating quantities depends on space and time through

$$
A(x, y, z, t)=A(z) \exp \left[i\left(k_{x} x+k_{y} y-\omega t\right)\right]
$$

If we consider a slowly varying background magnetic field, we expect that the system, which varies on times of the order of the helioseismic characteristic periods, has enough time to adapt the configuration corresponding to the instantaneous amplitude of the background magnetic field (adiabatic hypothesis). Under this assumption, by using a background magnetic field which varies in time according to $\boldsymbol{B}_{0}=B_{0} f\left(\omega_{0}\right) \boldsymbol{e}_{x}$, where $\omega_{0} \ll \omega$, we obtain two equations for the Fourier coefficients of magnetic field fluctuations $\boldsymbol{b}=\boldsymbol{B} /\left[B_{0} f\left(\omega_{0} t\right)\right]$ and the velocity fluctuations $v$

$$
\begin{aligned}
& -i \omega \boldsymbol{b}=i k_{x} \boldsymbol{v}-(\nabla \cdot \boldsymbol{v}) \boldsymbol{e}_{x} \\
& -i \omega \boldsymbol{v}=-\frac{\nabla P^{\prime}}{\rho_{0}}+\frac{\rho^{\prime} \boldsymbol{g}}{\rho_{0}}+v_{A}^{2}(t)\left[-\left(\frac{\partial b_{x}}{\partial z}-i k_{x} b_{z}\right)\right] \boldsymbol{e}_{x}
\end{aligned}
$$

("primed" quantities are fluctuations). The above set of equations is formally identical to that founded by Burgess 
et al. [31], apart for the fact that in our case

$$
v_{A}^{2}(t)=\frac{B_{0}^{2}}{4 \pi \rho} f^{2}\left(\omega_{0} t\right)
$$

is a time-dependent Alfvén speed.

After some algebraic calculations we finally obtain an equation for the fluctuating magnetic field, whose solution determines all other fluctuating quantities

$$
\begin{aligned}
& {\left[1-\frac{k_{x}^{2} v_{A}^{2}(t)}{\omega^{2}}\right] \frac{\mathrm{d}^{2} b_{z}}{\mathrm{~d} z^{2}}-\frac{N^{2}(z)}{g} \frac{\mathrm{d} b_{z}}{\mathrm{~d} z}} \\
& +k_{\perp}^{2}\left[\frac{k_{x}^{2} v_{A}^{2}(t)}{\omega^{2}}-1+\frac{N^{2}(z)}{\omega^{2}}\right] b_{z}=0
\end{aligned}
$$

where the perpendicular wave-vector is

$$
k_{\perp}^{2}=k_{x}^{2}+k_{y}^{2}
$$

and we defined the Brunt-Väisälä frequency

$$
N^{2}(z)=g(z)\left[\frac{1}{\gamma P_{0}} \frac{\mathrm{d} P_{0}}{\mathrm{~d} z}-\frac{1}{\rho_{0}} \frac{\mathrm{d} \rho_{0}}{\mathrm{~d} z}\right]
$$

which represents the characteristic frequency of the system. Equation (8) describes magneto-gravity waves. In the limit $B_{0} \rightarrow 0$ it leads to the standard helioseismic g-modes. In absence of gravity and $B_{0}=$ cost. Equation (8) describes Alfvén waves with frequency $\omega=\Omega_{A}=k_{x}$ $v_{A}$. By retaining both gravitation and magnetic field a new singular point occurs when the coefficient of the second derivative term in Equation (8) vanishes. Since this happens at $\omega=\Omega_{A}$, it can be viewed as being due to resonance between g-modes and Alfvén waves [31]. Let us come back to the Sun. Since $\Omega_{A}$ varies with the distance from the centre of the Sun, while, according to usual helioseismology the g-modes frequency is independent on position, the resonance occurs at a particular radius inside the Sun, namely when $\Omega_{A}$ crosses the frequency of one of the g-modes. The occurrence of the resonance depends on the value of $B_{0}$. This means that, in our case, the existence of the resonance is modulated in time by the term $f\left(\omega_{0} t\right)$, that is the resonance is time-dependent.

Solutions of Equations (7) gives the eigenvalue spectrum as roots of the trascendental equation [31]

$$
\chi=A(n, t) \cosh (\chi)
$$

where

$$
\omega=k_{x} C_{0}(t) \cosh (\chi)
$$

where $C_{0}(t)$ represents the time-dependent Alfvén velocity at the solar centre, and

$$
A(n, t)=\frac{k_{x} C_{0}(t)}{4 N k_{\perp} H}\left[2 \pi n-i \ln \left|\tan \frac{\pi}{\gamma}\right| \pm \pi\left(\frac{1-\gamma}{\gamma}\right)\right]
$$

Accordingly, the instantaneous resonant position is given by

$$
z_{r}(n, t)=2 H \ln \{\operatorname{Re}[\cosh \chi(n, t)]\}
$$

The time dependence of solution of Equation (10) results in a modulation of the distance between neighboring resonant layers with the same period of the background magnetic field. This is shown in Figure 3, where we report the time evolution of the distance as a function of the position of the resonance

$$
\mathrm{d} z_{r}(n, t)=z_{r}(n+1, t)-z_{r}(n, t)
$$

The background magnetic field is assumed to have a sinusoidal variation, with a profile defined by

$$
f\left(\omega_{0}, t\right)=\cos ^{2}\left(\frac{\omega_{0} t}{2}\right)+\varepsilon \sin ^{2}\left(\frac{\omega_{0} t}{2}\right)
$$

where $\omega_{0}=\pi y r^{-1}$ and the function $\mathrm{f}$ is defined in the interval $[\varepsilon, 1]$ (we used $\varepsilon=10^{-3}$ ).

As noted by Burgess et al. [31], for reasonably values of the background magnetic field intensity, the distance between resonant layers, at the neutrino's resonant region, are of the order of the neutrino's oscillation length. In particular the spikes which occur in density profiles, as a consequence of the resonance, could increase the probability of interactions between neutrino flux and solar matter [29].

In Figure 4 we report the time evolution of the lagrangian density perturbation

$$
\begin{aligned}
& \frac{\delta \rho}{\rho} \approx C \\
& -\left\{\exp \left[-\left(z-z_{r}(n, t)\right) / H\right](1+i 2 d(n, t))-1\right\}^{(1-\gamma) / \gamma}
\end{aligned}
$$

where $d(n, t)$ denotes the growing factor of the eigenfrequency $\omega=\omega_{1}(1+i d)$, and $\mathrm{C}$ is defined as follows

$$
C=\frac{\Gamma\left(\gamma^{-1}\right)}{\Gamma\left(-\gamma^{-1}\right)}\left[\frac{\gamma v_{p h}(n, t)}{c_{s} i \sqrt{\gamma-1}}\right]^{2 / \gamma}\left(\frac{\gamma-1}{4 \gamma}+\frac{\gamma^{2} k_{\perp}^{2} H^{2}}{\gamma-1}\right)
$$

where $v_{p h}(n, t)=\omega_{1}(n, t) / k_{x}$. As it is evident, the resonance oscillates in time with a frequency $\omega_{0}$.

\section{Conclusions}

Recent analysis carried on BiSON and GOLF data [40] show that quasi-biennial signal has the same amplitude for p-modes at all frequencies. On the other hand the 11 -yr modulation affects predominantly high frequency p-modes occuring on shallow regions close to the solar surface. This suggests that the dynamo mechanism responsible of the mean cycle has its origin at shallow regions of the solar interior (resonably located near the bottom of the shear layer extending 5\% below the surface), 


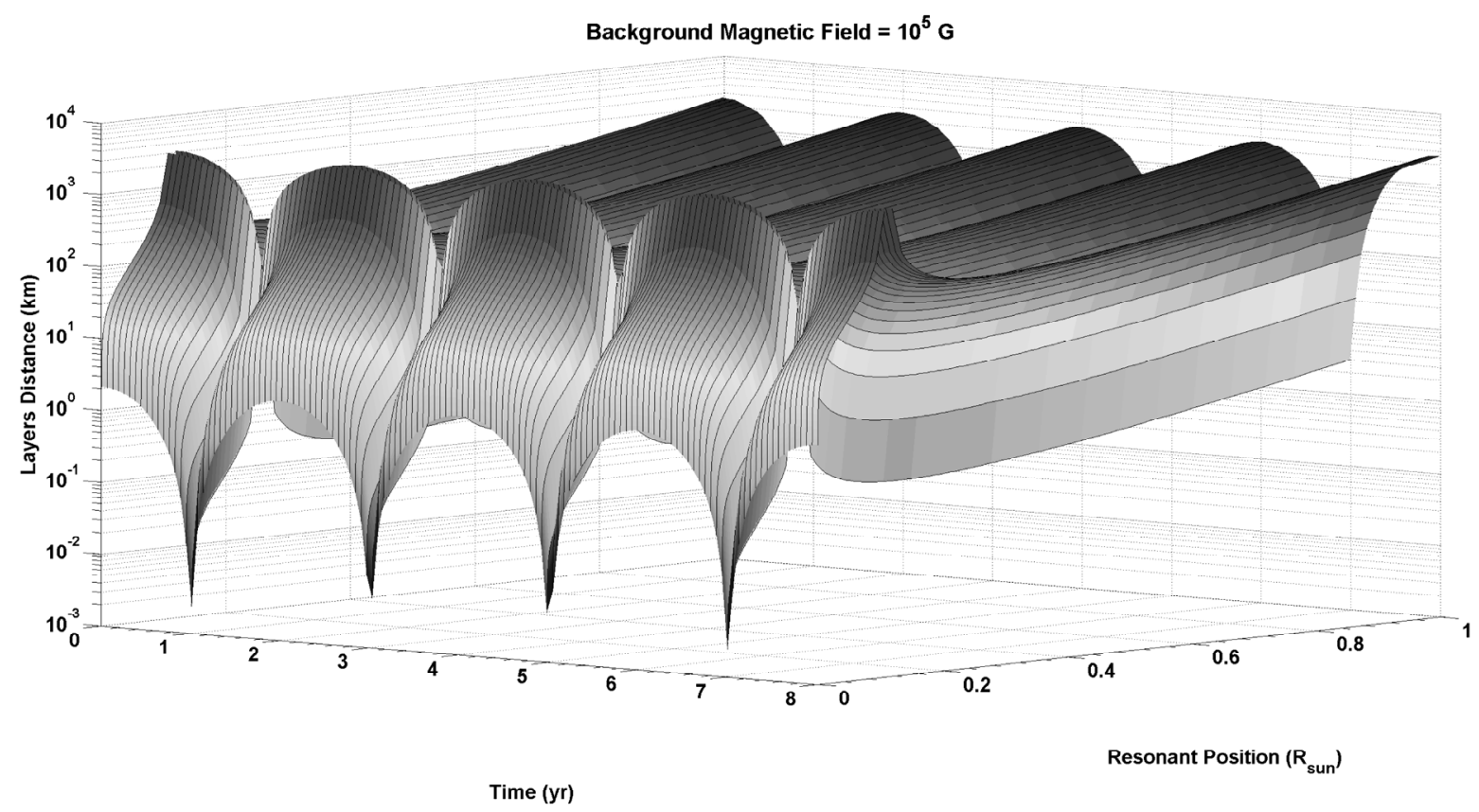

Figure 3. Time evolution of the distance between resonant Alfvén layers. In the $y$-z plane are reproduced respectively the position of the resonances (in solar radius units) and the distance between the resonant layers (in $\mathrm{km}$ ). The $x$ axis represents the time (in yr).
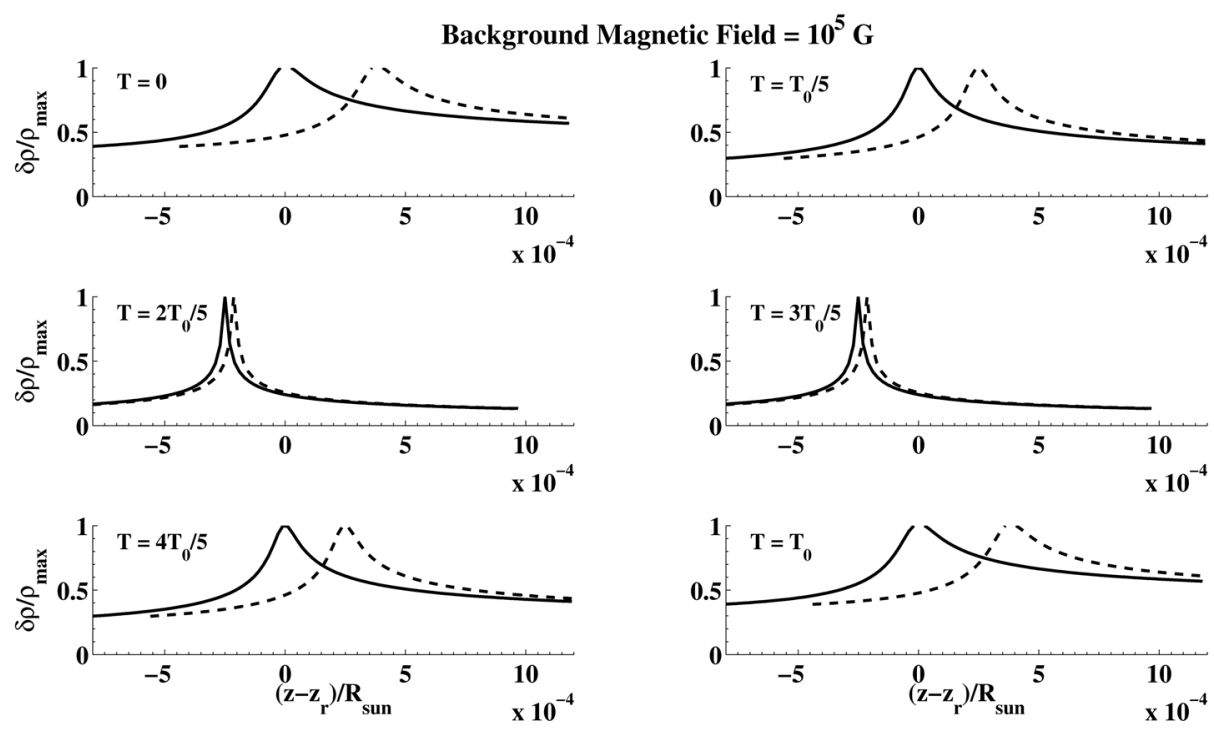

Figure 4. Time evolution of neighbouring density profiles in the region $z_{r} \sim 0.3 R_{\text {sun }}$.

while another separated quasi-biennial dynamo mechanism could be originated in deeper layers. In this scenario, the quasi-biennial dynamo located in the inner layers of the Sun, is more likely to induce a fluctuating background magnetic field. The latter is the key ingredient of the model since allows that correlation length between density spikes variates in time. This mechanism could thus produce the observed variations, at the quasibiennial scale, of the solar neutrino flux.

The modulation of solar neutrinos and the coupling with magneto-gravity modes is of great interest for solar physics. This coupling could represent a new way to investigate the physical properties in the very inner layers of the Sun thus playing the role of a "telescope" for the solar interior.

\section{Acknowledgements}

This work was partially supported by the ASI/INAF Contract No. I/022/10/0, by the European Social Fund- 
European Commission and Regione Calabria. Thanks are due also to the Italian PNRA for the use of the RACANT database prepared at IFSI-Roma.

\section{REFERENCES}

[1] R. Davis Jr., D. S. Harmer and K. C. Hoffman, "Search for Neutrinos from the Sun," Physical Review Letters, Vol. 20, No. 21, 1968, pp. 1205-1209. doi:10.1103/PhysRevLett.20.1205

[2] W. R. Sheldon, "Possible Relation of a Null Solar Neutrino Flux to the II Year Solar Cycle," Nature, Vol. 221, No. 5181, 1969, pp. 650-651. doi:10.1038/221650b0

[3] R. A. Donahue and S. L. Baliunas, "Periodogram Analysis of 240 Years of Sunspot Records," Solar Physics, Vol. 141, No. 1, 1992, pp. 181-197. doi:10.1007/BF00155911

[4] Ya. B. Zeldovich, A. A. Ruzmaikin and D. D. Sokoloff, "Magnetic Fields in Astrophysics," Gordon \& Breach, New York, 1983.

[5] A. Pontieri, F. Lepreti, L. Sorriso-Valvo, A. Vecchio and V. Carbone, "A Simple Model for the Solar Cycle," Solar Physics, Vol. 213, No. 1, 2003, pp. 195-201. doi:10.1023/A:1023227503176

[6] K. Sakurai, "Quasi-Biennial Variation of the Solar Neutrino Flux and Solar Activity," Nature, Vol. 278, 1979, pp. 146-148. doi:10.1038/278146a0

[7] L. J. Lanzerotti and R. S. Raghavan, "Solar Activity and Solar Neutrino Flux," Nature, Vol. 293, 1981, pp. 122124. doi:10.1038/293122a0

[8] Y. Fukuda, et al., (Kamiokande Collaboration), "Solar Neutrino Data Covering Solar Cycle 22," Physical Review Letters, Vol. 77, No. 9, 1996, pp. 1683-1686. doi:10.1103/PhysRevLett.77.1683

[9] G. A. Bazilevskaya, M. B. Krainev, V. S. Makhmutov, E. O. Flückiger, A. I. Sladkova and M. Storini, "Structure of the Maximum Phase of Solar Cycles 21 and 22," Solar Physics, Vol. 197, No. 1, 2000, pp. 157-174. doi:10.1023/A:1026515520311

[10] A. Vecchio and V. Carbone, "On the Origin of the Double Magnetic Cycle of the Sun," The Astrophysical Journal, Vol. 683, No. 1, 2008, pp. 536-541. doi: $10.1086 / 589768$

[11] J. F. Valdés-Galicia and V. M. Velasco, "Variations of Mid-Term Periodicities in Solar Activity Physical Phenomena," Advances in Space Research, Vol. 41, No. 2, 2008, pp. 297-305. doi:10.1016/j.asr.2007.02.012

[12] A. Vecchio, M. Laurenza, V. Carbone and M. Storini, "Quasi-Biennial Modulation of Solar Neutrino Flux and Solar and Galactic Cosmic Rays by Solar Cyclic Activity," The Astrophysical Journal, Vol. 709, No. 1, 2010, pp. L1-L5. doi:10.1088/2041-8205/709/1/L1

[13] A. Vecchio and V. Carbone, "Spatio-Temporal Analysis of Solar Activity: Main Periodicities and Period Length Variations," Astronomy and Astrophysics, Vol. 502, No. 3, 2009, pp. 981-987. doi:10.1051/0004-6361/200811024

[14] J. F. Valdés-Galicia, R. Pérez-Enríquez and J. A. Otaola, "The Cosmic-Ray 1.68-Year Variation: A Clue to Under- stand the Nature of the Solar Cycle?" Solar Physics, Vol. 167, No. 1-2, 1996, pp. 409-417.

doi:10.1007/BF00146349

[15] K. Mursula and J. H. Vilpolla, "Fluctuations of the Solar Dinamo Observed in the Solar Wind and Interplanetary Magnetic Field at 1 AU and in the Outer Heliosphere," Solar Physics, Vol. 221, No. 2, 2004, pp. 337-349. doi:10.1023/B:SOLA.0000035053.17913.26

[16] M. Laurenza and M. Storini, "Interpretation of Quasi Periodic Variations in Solar Cosmic Ray Data," Proceedings of the 31st ICRC, ŁÓDŹ, 2009.

[17] M. Laurenza, A. Vecchio, V. Carbone and M. Storini, "Quasi Biennial Modulation of Galactic Cosmic Rays," The Astrophysical Journal, 2012 (in press).

[18] M. Laurenza, M. Storini, S. Giangravé and G. Moreno, "Search for Periodicities in the IMP8 Charged Particle Measurement Experiment Proton Fluxes for the Energy Bands 0.50 - $0.96 \mathrm{MeV}$ and 190 - $440 \mathrm{MeV}$," Journal of Geophysical Research, Vol. 114, No. 1A, 2009. doi:10.1029/2008JA013181

[19] J. Javaraiah, R. K. Ulrich, L. Bertello and J. E. Boyden, "Search for Short-Term Periodicities in the Sun's Surface Rotation: A Revisit," Solar Physics, Vol. 257, No. 1, 2009, pp. 61-69. doi:10.1007/s11207-009-9342-9

[20] R. Davis Jr. and J. C. Evans, "Experimental Limits on Extraterrestrial Sources of Neutrinos," Proceeding of 13 th International Conference on Cosmic Rays, Vol. 3, 1973, pp. 2001-2006.

[21] Y. Fukuda, et al., "Measurements of the Solar Neutrino Flux from Super-Kamiokande's First 300 Days," Physical Review Letters, Vol. 81, No. 6, 1998, pp. 1158-1162. doi:10.1103/PhysRevLett.81.1158

[22] L. M. Krauss, "Correlation of Solar Neutrino Modulation with Solar Cycle Variation in $p$-Mode Acoustic Spectra," Nature, Vol. 348, No. 6300, 1990, pp. 403-407. doi: $10.1038 / 348403 \mathrm{a} 0$

[23] J. N. Bahcall and W. H. Press, "Solar-Cycle Modulation of Event Rates in the Chlorine Solar Neutrino Experiment," The Astrophysical Journal, Vol. 370, 1991, pp. 730-742. doi:10.1086/169856

[24] D. S. Oakley, H. B. Snodgrass, R. K. Ulrich and T. L. Vandekop, "On the correlation of solar surface magnetic flux with solar neutrino capture rate," The Astrophysical Journal, Vol. 437, No. 1, 1994, pp. L63-L66. doi: $10.1086 / 187683$

[25] R. L. McNutt Jr., "Correlated Variations in the Solar Neutrino Flux and the Solar Wind and the Relation to the Solar Neutrino Problem," Science, Vol. 270, No. 5242, 1995, pp. 1635-1639. doi:10.1126/science.270.5242.1635

[26] R. M. Wilson, "Correlative Aspects of the Solar Electron Neutrino Flux and Solar Activity," The Astrophysical Journal, Vol. 545, No. 1, 2000, pp. 532-546. doi: $10.1086 / 317787$

[27] P. A. Sturrock, "Solar Neutrino Variability and Its Implications for Solar Physics and Neutrino Physics," The Astrophysical Journal Letters, Vol. 688, No. 1, 2008, pp. L53-L56. doi:10.1086/594993

[28] O. G. Miranda, T. I. Rashba, A. I. Rez and J. W. F. Valle, 
"Enhanced Solar Antineutrino Flux in Random Magnetic Fields," Physical Review D, Vol. 70, No. 11, 2004, Article ID: 113002. doi:10.1103/PhysRevD.70.113002

[29] P. Bamert, C. P. Burgess and D. Michaud, "Neutrino Propagation through Helioseismic Waves," Nuclear Physics B, Vol. 513, No. 1-2, 1998, pp. 319-342. doi:10.1016/S0550-3213(97)00672-X

[30] C. P. Burgess, N. S. Dzhalilov, M. Maltoni, T. I. Rashba, V. B. Semikoz, M. A. Tórtola and J. W. F. Valle, "Large Mixing Angle Oscillations as a Probe of the Deep Solar Interior," The Astrophysical Journal Letters, Vol. 588, No. 1, 2003, pp. L65-L68. doi:10.1086/375482

[31] C. P. Burgess, N. S. Dzhalilov, T. I. Rashba, V. B. Semikoz and J. W. F. Valle, "Resonant Origin for Density Fluctuations Deep within the Sun: Helioseismology and Magneto-Gravity Waves," Monthly Notices of the Royal Astronomical Society, Vol. 348, No. 2, 2004, pp. 609-624. doi:10.1111/j.1365-2966.2004.07392.x

[32] A. Vecchio, L. D’Alessi, V. Carbone, M. Laurenza and M. Storini, "The Empirical Mode Decomposition to Study the Quasi-Biennial Modulation of Solar Magnetic Activity and Solar Neutrino Flux," Advances in Adaptive Data Analysis, Vol. 4, No. 1-2, 2012, Article ID: 1250014. doi:10.1142/S1793536912500148

[33] L. D'Alessi, A. Vecchio, M. Laurenza, M. Storini and V. Carbone, "Solar Neutrino Flux Modulated by Solar Activity," Proceeding of the International School of Physics "E. fermi", Vol. 182: Neutrino Physics and Astrophysics, IOS Press, Amsterdam, 2012, pp. 349-351.

[34] R. Davis, Private Communications, 2004.

[35] J. N. Abdurashitov, et al., (SAGE Collaboration), "Meas- urement of the Solar Neutrino Capture Rate with Gallium Metal. III. Results for the 2002-2007 Data Tacking Period," Physical Review C, Vol. 80, No. 1, 2009, Article ID: 015807, pp. 1-16. doi:10.1103/PhysRevC.80.015807

[36] N. E. Huang, Z. Shen, S. R. Long, M. C. Wu, H. H. Shih, Q. Zheng, N. C. Yen, C. C. Tung and H. H. Liu, "The Empirical Mode Decomposition and the Hilbert Spectrum for Nonlinear and Non-Stationary Time Series Analysis," Proceedings of the Royal Society A, Vol. 454, 1998, pp. 903-995. doi:10.1098/rspa.1998.0193

[37] D. A. T. Cummings, R. A. Irizarry, N. E. Huang, T. P. Endy, A. Nisalak, K. Ungchusak and D. S. Burke, "Travelling Waves in the Occurence of Dengue Haemorrhagic Fever in Thailand," Nature, Vol. 427, 2004, pp. 344-347. doi: $10.1038 /$ nature 02225

[38] J. Terradas, R. Oliver and J. L. Ballester, "Application of Statistical Techniques to the Analysis of Solar Coronal Oscillations," The Astrophysical Journal, Vol. 614, No. 1, 2004, pp. 435-447. doi:10.1086/423332

[39] D. M. Simpson, A. F. C. Infantosi and D. A. BoteroRosas, "Estimation and Significance Testing of CrossCorrelation between Cerebral Blood Flow Velocity and Background Electro-Encephalograph Activity in Signals with Missing Samples," Medical and Biological Engineering and Computing, Vol. 39, No. 4, 2001, pp. 428433. doi:10.1007/BF02345364

[40] S. T. Fletcher, A. M. Broomhall, D. Salabert, S. Basu, W. J. Chaplin, Y. Elsworth, R. A. Garcia and R. New, "A Seismic Signature of a Second Dinamo?" The Astrophysical Journal Letters, Vol. 718, No. 1, 2010, pp. L19-L22. doi:10.1088/2041-8205/718/1/L19 